\title{
Modeling the Local Bubble
}

\author{
Donald P. Cox \\ Department of Physics, University of Wisconsin-Madison \\ 1150 University Ave., Madison, WI 53706 USA \\ cox@wisp.physics.wisc.edu
}

\begin{abstract}
Modeling the Local Bubble is one of those activities fraught with danger. It is very easy to be too naive, to fail to consider the dependence of the model on assumptions about the nearby ambient state, or the likelihood of such a structure. It is similarly easy to become so caught up in the details of the vicinity that it is unclear where to begin a necessarily idealized modeling effort. And finally, it is important to remember that the data we have may in some cases be lying to us, and that we have not yet learned to read their facial expressions quite carefully enough.

That said, I've tried in this paper to be helpful to those who may wish to take the risks. I surveyed the very most basic stories that the data seem to tell, and pointed out the standard coincidences that may be telling us a lot about what is happening, but may turn out once again to have been just coincidences. I've described 5 distinct conceptions that in one flavor or another pretty well survey the collection of mental images that have so far been carried by those who've attempted models. One may be right, or something entirely different may be more appropriate. It's at least vital to realize that a conception comes first, followed by a simplified model of details. I've also included a long list of questions directed at observers. Some have partial answers, some one wouldn't know today quite how to approach. But it is a list that students of the soft $\mathrm{x}$-ray background, interstellar absorption lines, possible instrumentation, and the heliosphere may wish to review from time to time, just to see whether they can figure out how to be more helpful. There is another list for modelers, things the models must address, however-so-flimsily if necessary, because there are strong observational constraints (and stronger ones coming) on what can and cannot be present in the local ISM. To that I've added a few remarks concerning $x$-ray emission coming from beyond the Local Bubble, and another few on how x-ray emission from within the solar system might be contaminating what we see. That last bit is new, exciting, and possibly wrong, but it is an example of the ongoing wariness I believe one has to take toward the facts in the case. By the way, Dieter, it really was a great meeting.
\end{abstract}

\section{The Challenge}

The fundamental challenge in modeling the Local Bubble is to find a source for approximately 200 solar luminosities of diffusely distributed soft x-ray radiation in a volume containing roughly a million stars.

One possibility is that there is million Kelvin gas at normal interstellar pressure filling an irregular volume of roughly $100 \mathrm{pc}$ extent. The thermal 
radiation from the optically thin gas would then supply the emission. The cooling time of the hot gas has been estimated at about $2 \times 10^{7}$ years and one presumes that the age and lifetime of the bubble is some modest fraction of that. Assuming this configuration, modelers then concentrate on how the low density region might have come into being, what sort of boundary it has, what reheated the region most recently, when that occurred, and whether it is likely to occur again before the region's demise. (A volume of radius 100 pc probably has one or more random supernovae within it during a bubble lifetime.) Cox and Reynolds (1987) present a review.

But there may be other possibilities. One looks for continuous inputs that might keep the gas hot, perhaps leading to a steady-state phase of hot gas whenever the density gets low (H. J. Habing, 1969, private communication). Solar type stellar winds inject energy with sufficient vigor, but far too many would seem to be required. Consider however, the following coincidence: The Galactic energy flux in cosmic rays in the solar neighborhood is about 1 to $2 \times 10^{-5} \mathrm{erg} \mathrm{cm}^{-2} \mathrm{~s}^{-1}$, providing about 1000 to 2000 solar luminosities from an area of radius $100 \mathrm{pc}$. The escape time of the cosmic rays is about $2 \times 10^{7}$ years. Within the uncertainties, the (local) power requirements and timescales of the Local Bubble and cosmic rays are comparable, though there is no obvious connection.

\section{Three More Coincidences}

The intensity and broad band "colors" of the x-ray emission, interpreted with a plasma radiation code, yield measures of the temperature $T$ and emission measure $n_{e}^{2} R$ of the diffuse hot gas in the Local Bubble. Combining that with stellar absorption measures of the extent of the low density region around the Sun, to get $R$, yields an rms electron density measure and thus a pressure estimate. Knowledge of the volume and pressure allow us to estimate the thermal energy content of the bubble. Knowledge of the temperature and radial scale provide a rough estimate of the thermal conduction flux from the bubble to its boundary. From this exercise, three coincidences appear that illuminate and possibly confound modeling of the bubble: 1) The thermal pressure in the bubble is approximately the weight per unit area of the interstellar medium, the total interstellar average pressure at midplane, a factor of 3 to 10 higher than thermal pressures in clouds. 2) The thermal energy content of the bubble is about the energy of 1 supernova. 3) The possible thermal conduction flux to the edge, about $10^{-6}\left(T / 10^{6} \mathrm{~K}\right)^{7 / 2}(100 \mathrm{pc} / R)$ erg $\mathrm{cm}^{-2} \mathrm{~s}^{-1}$, is approximately the same as the thermal radiation flux inferred from the $\mathrm{x}$-ray emission.

Although the extreme sensitivity to temperature makes it difficult to insist that the third coincidence is meaningful, it is possible to argue that an evolving hot bubble would tend to this state. If one supernova worth of energy occupies a large bubble at normal interstellar pressure, its radius will 
be of order $100 \mathrm{pc}$. If it has a temperature well in excess of $10^{6} \mathrm{~K}$, thermal conduction will evaporate material from the surroundings, raising its density and lowering its temperature, until the conduction flux is just able to be radiated by the denser cooler material in the outer parts of the bubble. At that point its temperature will be of order $10^{6} \mathrm{~K}$ and its lifetime about $10^{7}$ years. In short, apart from the problem of creating the low density region in the first place, what we see is about what one might expect.

Except that what one might expect depends fundamentally on one's preconceptions of conditions in the ambient ISM, and the physical processes which are important. Is the galactic disk mostly at very low density and fairly hot, is it mostly warm intercloud gas, do clouds thermally evaporate when surrounded by hot gas, is thermal conduction active within the hot gas or quenched by a tangled magnetic field, do motions within the ambient medium so strongly distort a bubble in $10^{7}$ years that it is meaningless to consider such lifetimes? The ambient ISM is far from understood, making it very difficult to know how to model the Local Bubble as an event within it (see Cox 1996a,b for thoughts on modeling the general ISM).

\section{Four Conceptions, Maybe Five}

At the meeting, I described what I thought were the four distinct views of the Local Bubble on which models have been based. For completeness, I now think it is important to include a fifth view.

\subsection{The Hot Phase Model}

In this model, much of interstellar space is assumed to be filled with million Kelvin gas at normal interstellar pressure, but our ability to see beyond 100 pc or so is limited by the local distribution of denser gas which readily absorbs the soft $x$-rays. The view was originally proposed by Cox and Smith (1974) and later explored in some detail by Jakobsen and Kahn (1986) and Kahn and Jakobsen (1988). In this visibility bounded view, there is no distinct Local Bubble, only the parts of the hot phase we are able to see from the solar location.

\subsection{An Active SNR in an Already Low Density}

If the ambient interstellar density in our vicinity were about $0.01 \mathrm{~cm}^{-3}$, a single supernova remnant with an age of about $10^{5}$ years, with the Sun inside, would resemble what we see. This differs substantially from the more quiescent bubble picture developed from the coincidences above (and modeled as an old Multi-SNR below) in that there is still a strong shock at the edge, heating gas to $10^{6} \mathrm{~K}$; the $\mathrm{x}$-ray emitting gas is recently heated, underionized, and moving radially outward at about $300 \mathrm{~km} \mathrm{~s}^{-1}$. This scenario 
was originally proposed by McKee and Ostriker (1977) within the context of their SNR dominated ISM model as a means of achieving x-ray emitting temperature gas locally, their hot phase being generally too cool for $\mathrm{x}$-ray emission. It has been modeled in various versions since by Cox and Anderson (1982), Arnaud et al. (1984), and Edgar (1986).

\subsection{An Old Multi-SNR}

If the ambient interstellar density is closer to $0.1 \mathrm{~cm}^{-3}$, a sequence of supernovae in the same region is required to build the low density cavity to the necessary size and then to reheat the interior. Once the most recent explosion's shock has reached the bubble boundary and reverberated a time or two, the evolution is slow and closely approximates a quiescent bubble of cooling hot gas. If thermal conduction is not severely quenched and the ambient medium not too active, the bubble tends toward a structure consistent with the observed coincidences. This scenario is similar to the development of a superbubble except that the recurring supernovae are infrequent (at perhaps one to a few million year intervals), only a few in total number, and the most recent is long enough in the past that it has lost its separate identity. The overall pressure in the bubble is comparable to that in the ambient medium. Any shell has long since rebounded to ambient density; the velocity of either expansion (if relatively young) or contraction (if older) is small, about $10 \mathrm{~km}$ $\mathrm{s}^{-1}$. The idea for such a scenario derived from a single old quiescent SNR model by Innes and Hartquist (1984), and has been explored further by Cox and Snowden (1986), Cox and Reynolds (1987), Edgar and Cox (1993) and finally modeled in hydrodynamics and ionization with a sequence of explosions by Smith (1995) and presented in this volume by Smith and Cox (1997). Come to think of it, the single old quiescent SNR (in an already low density region) model of Innes and Hartquist is a sixth conception, in addition to having motivated the multi-SNR one.

\subsection{An Exploded Cloud}

In this scenario, roughly 10 supernovae occur very close together in time in a high density cloud, either close to the boundary or in a small cloud. The supernovae heat the cloud to very high temperature and pressure and it expands freely into a much lower density environment. The expansion lowers the temperature dramatically but the high ionization is frozen in. The expansion is subsequently slowed by the surroundings, but is dense enough that any reheating is transient. The present remnant is cool gas at moderately low density filling the local region. Its electron density is the same as the average in the ISM, explaining the dispersion measures of nearby pulsars, its thermal pressure is similar to that of the Local Cloud, its EUV emission is very low, and its $\mathrm{x}$-rays arise from the recombination of highly ionized heavy elements (Breitschwerdt and Schmutzler, 1994). 


\subsection{Part of the Sco-Cen Association Superbubble}

Noticing that Loop I is probably the edge of the Sco-Cen superbubble, that it occupies a very large angular size on the sky, and that the filaments of Local Fluff passing over the Solar System have velocities similar to that of the approaching hydrogen between us and the Sco-Cen Association leads to a different view. This scenario proposes that the Local Bubble is actually part of the Sco-Cen superbubble, perhaps preferentially expanded in a low density (interarm) direction. This view has been taken by Bochkarev (1987) and Frisch (e.g. this volume and references therein). It is not entirely clear how this scenario leads to hot gas surrounding the solar location while there is still a dense wall of neutral hydrogen about $70 \mathrm{pc}$ away, between us and the Sco-Cen Association. No detailed model has been made, but the scenario is sufficiently complicated (read: realistic) that it would be difficult to imagine how to go about it. There seem to be two important aspects of this view, one that the Sco-Cen Association offers the only obvious nearby source of bubble power, there being no O or B stars in the Local Bubble itself. The other is the realization that the Local Bubble environment is far from simple; if nothing else it is at least partly wrapped around or tangent to the Loop I bubble, with possible free connections between their hot gas contents at high latitude (Cox and Reynolds, 1987).

This ends my survey of conceptions. The list is heavily tilted toward supernovae as the causative agent, but it may turn out that they were only the easiest thing to imagine or model.

\section{Background and Observational Issues}

There are many questions that need to be addressed observationally before we can have a confident interpretation of the Local Bubble. They include:

- Do the x-rays observed in the galactic plane definitely arise from outside the solar system?

- Do the x-rays have the spectral features of hot gas, or of recombining highly ionized cool gas, or is much of it an unexplained continuum?

- The gas phase elemental abundances in the hot gas, are they solar, depleted, or enriched?

- Is there evidence of edge brightening, suggesting emission concentrated at the outer surface rather than spread through the volume?

- Are there any regions of spectral anomaly that might suggest interaction between the Local Bubble and the hot gas of Loop I?

- Do spectral maps support the conclusion from broad band surveys that the Local Bubble is emission-bounded rather than visibility-bounded?

- Is there fast moving or hot O VI associated with the Local Bubble? Is the $\mathrm{O}$ VI associated with the bubble boundary consistent with quiescent hot bubble models? 
- Is there any hint of boundary structure between the hot gas and the Local Cloud? Is the cloud evaporating and if so, how fast compared to the classical rate?

- In directions in which the hot gas appears to butt up against dense background material, what is the nature of the interaction? Is there any sign of evaporation or condensation? How do the high stage ions found in absorption compare with the x-ray spectral characteristics?

- Is there a region in which we can see the hot gas tangentially along a boundary, and if so does the spectral gradient confirm the conductive condensation picture?

- What more can we learn about regions of bizarre emptiness such as that toward epsilon CMa?

\section{Issues Which Models Must Confront}

A responsible attempt to model the Local Bubble should confront at least the following issues:

- What is the assumed environment prior to the generation of the Local Bubble and is that consistent with general ISM constraints?

- How likely is the Solar System to be found in a region of the sort envisioned? This is the Copernican question and is addressed by specification of the generating agent, estimation of the rate of creation of such regions and their lifetimes.

- How does the model produce the requisite x-ray surface brightness, the band ratios, and, when the long awaited observations are made, the spectrum?

- Is the model consistent with the degree of elemental depletion onto dust (or elemental enrichment) that will be measured from that $\mathrm{x}$-ray spectrum?

- Is the model consistent with the strong limit on slow narrow O VI in the local vicinity and the $O$ VI column densities and velocity profiles associated with the Local Bubble that should be available from FUSE?

- How does the model address the apparent displacement effects (the xray- $\mathrm{NH}$ anticorrelation): the sign of the effect, the color independence, the implication that the emission is in the volume rather than on the surface.

- How is the model consistent with the existence and persistence of the Local Fluff? 


\section{Three Remarks on X-Ray Emission from Beyond the Local Bubble}

\subsection{Breitschwerdt and Schmutzler's Wind Model}

Breitschwerdt and Schmutzler (1994) also proposed a model for generation of high latitude $\mathrm{x}$-ray emission from a galactic wind, the material initially having been injected by superbubbles into channels or chimneys leading out through the halo. A novel feature of their proposal was that some amount of the $x$-ray emission derived, as in their Local Bubble model, from recombination of high stage ions in expansion-cooled gas.

But, there is a simple upper limit to the amount of x-ray power available from delayed recombination. Each hydrogen atom escaping in the wind is accompanied by about 0.001 heavy ions capable of making $x$-rays in their recombination. Each of those ions can produce about $1 \mathrm{keV}$ of $\mathrm{x}$-rays in their recombination, for a total of about $2 \times 10^{-12} \mathrm{ergs}$ per hydrogen atom. In their model, 0.4 solar masses of material per year left in the wind, $4 \times 10^{56}$ atoms per year, for a total recombination power of $10^{45}$ ergs per year, about $0.01 \%$ of the Galaxy's supernova power or $1 \%$ of the power density of the soft $\mathrm{x}$ ray background. The bulk of the x-ray emission in their wind model is from thermal excitation of emission lines prior to the flow's cooling below roughly $10^{6} \mathrm{~K}$.

\subsection{Halo Supernovae}

There have been a variety of potential sources suggested for high latitude high stage ions and x-ray emission: a galactic wind, shocked diffuse galactic infall, wakes of infalling clouds, a galactic chromosphere, a quiescent galactic corona (e.g. Wang, this volume), microflare reconnection events, a general galactic fountain arising from a hot phase of the ISM, localized plumes arising through superbubble chimneys, etc. I would like to call attention to yet another potential contributor, halo supernovae, whose modeling has recently been undertaken by Robin Shelton (this volume).

\subsection{The ROSAT Distant Component}

It is my understanding that there are two ROSAT distant components, a hard one which seems to be concentrated around the galactic center and discussed in this volume by Wang and by Freyberg, and a softer one explored at moderately high latitude by shadowing experiments and described in this volume by Snowden. My comments are directed at the latter.

A reader familiar, for example, with the Cox and Reynolds (1987) review of the Local ISM will notice that my opinions concerning the characteristics, structure, and origin of the Local Bubble have evolved very little in the intervening years. The reason is found in Snowden's summary paper: ROSAT 
showed us a great deal about emission in the low lying halo, beyond the Local Bubble, but did little to alter our understanding of the bubble itself. A smaller fraction of the observed emission from high latitude is local, but the part that is local still has pretty much the original description.

But what is the "halo emission"? From Snowden et al. (1997), most of the excess emission is very nonuniform; it lies close to the galactic plane, and it tends to be found above active regions in the disk. My tentative summary is that it arises in discrete large scale regions in the thick disk of interstellar material, powered by events in the underlying thin disk. Referring to these very bright features as "halo emission" obscures the possibility that there may be a truly diffuse component at lower intensity that better deserves the name.

\section{Solar System X-Ray Emission}

In the early days of soft x-ray background studies, we tried very hard at Wisconsin to think of ways that a substantial $x$-ray contribution could be made within the solar system. We concerned ouselves with scattered solar $\mathrm{x}$-rays, with colliding fast streams in the solar wind, with solar wind impacts on zodiacal dust, with the solar wind termination shock and the solar wind's magnetotail, and probably others I have since forgotten. We eventually concluded that because we could think of no major contributing processes there were none.

That, however, was before the discovery of "Long Term Enhancements" and the intense $\mathrm{x}$-ray emission of comets!

Long Term Enhancements are the name given, I believe by Steve Snowden, to a time-variable component of the diffuse emission seen by ROSAT. It was a form of contamination that seemed uniform to within a factor of two over a great circle perpedicular to the Earth-Sun line, varying on a timescale of tens of hours. It looks just like the C-band x-rays from the sky, may come from closer than the moon (according to Snowden, private communication, it may have produced the dark side counts seen in the ROSAT picture of the moon), correlates with practically nothing and has an unknown origin. If the process producing this "emission" has a DC component, that component has not been subtracted from the soft x-ray background and would have been attributed to the Local Bubble. If such contamination were all of the emission seen in the galactic plane, only the higher latitude emission would be galactic and the Local Bubble would be two, one each above and below us in $z$. I do not offer these worries as a serious concern; the contribution would have had to remain stable for many years and been independent of observing instrument. I am concerned, however, that there is an undiscovered local process that can generate such contamination.

And along came Hyakutake, blazing away at $10^{-5} \mathrm{ergs}^{-2} \mathrm{sm}^{-1}$, com-

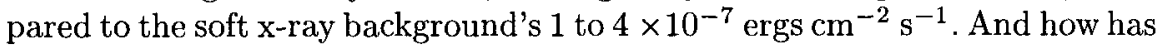


that been interpreted? Why, with the Breitschwerdt and Schmutzler mechanism! Or at least something similar. The solar wind has a particle flux of roughly $2 \times 10^{8}(1 \mathrm{AU} / r)^{2}$ atoms $\mathrm{cm}^{-2} \mathrm{~s}^{-1}$, with an average heavy element ionization energy of about $2 \times 10^{-12}$ ergs per particle, making the potential $\mathrm{x}$-ray recombination flux about $4 \times 10^{-4}(1 \mathrm{AU} / r)^{2} \mathrm{ergs} \mathrm{cm}^{-2} \mathrm{~s}^{-1}$. By impacting the comet's halo, the solar wind encounters enough material to force a substantial fraction of the high stage ions to recombine, producing the observed flux (Cravens, 1997; Haberli, Gombosi, De Zeeuw, Combi, and Powell, 1997). It would be nice to have the confirming spectrum, but the mechanism is exceedingly plausible.

Could the same mechanism be responsible for the Long Term Enhancements, or to a DC level in the soft x-ray background? If all of the high stage ions in the solar wind are forced into recombination prior to reaching about 60 $\mathrm{AU}$, their $\mathrm{x}$-ray flux will be significant. (If a small fraction recombine closer, that too is significant.)

One would expect interplanetary transient events to be more localized in the sky than the LTEs, to have the wrong timescale, and not to be produced closer than the moon; but LTEs could potentially arise in interaction between the solar wind and the exospheric hydrogen of the geocorona. For the most part these two do not intermix, but the required efficiency is only 0.001 . Above $10,000 \mathrm{~km}$ altitude $h$, the geocoronal density is roughly $2 \times 10^{3}\left(10^{4} \mathrm{~km} / h\right)^{2}$ $\mathrm{cm}^{-3}$ (e.g. He et al., 1993). With the solar wind flux given above and a charge exchange cross section of $4 \times 10^{-15} \mathrm{~cm}^{2}$, the line of sight integrated $\mathrm{x}$-ray emissivity from beyond 10 Earth radii would be of order $5 \times 10^{-7} \mathrm{ergs}$ $\mathrm{cm}^{-2} \mathrm{~s}^{-1}$, easily comparable to the SXRB. Very likely the required 0.001 efficiency could occur via a variety of complications, but it is surprising that even this zeroth order estimate is of the required magnitude.

With this mechanism, one would expect the LTE intensity to depend on the current solar wind particle flux and ionization level, the exospheric hydrogen density profile, the relative orientation between the Sun-satellitelocation-look-direction, and the degree to which the magnetospheric boundary allows solar wind access to the geocoronal hydrogen. The complexity of this dependency may have hidden it from correlations examined so far.

A DC level could potentially arise in interaction of the solar wind with the stream of interstellar material flowing through the Solar System. Assuming the interstellar stream density is roughly constant beyond $10 \mathrm{AU}$ (within which it gets photoionized) at $0.1 \mathrm{~cm}^{-3}$, and a charge exchange cross section of order $4 \times 10^{-15} \mathrm{~cm}^{2}$ yields a recombination probability of $5 \times 10^{-3}$ per $\mathrm{AU}$. Integrating the implied emissivity from $10 \mathrm{AU}$ out yields an estimated $\mathrm{x}$-ray flux of $2 \times 10^{-7} \mathrm{ergs} \mathrm{cm}^{-2} \mathrm{~s}^{-1}$ for the upwind direction. As this is the observed level of emission in the soft $x$-ray background, it would appear that such a calculation needs to be made more carefully; it could well turn out that the spectrum of the soft $\mathrm{x}$-ray background is locally contaminated with a significant recombination flux. 
Should we be worried? Well, yes and no. The recombination spectrum calculated by Haberli et al. shows very little emission in the carbon band and could be ruled out as a major contaminant of the SXRB from even the crude pulse height distributions of the Wisconsin rocket data. In addition, there is a strong oxygen line predicted at $574 \mathrm{eV}$ that would appear in the $M$ band where there is no Long Term Enhancement and where shadowing experiments seem to show that the preponderance of the emission is quite distant. On the other hand, carbon band emission is expected to arise from silicon, sulfur, magnesium, and iron-elements that were simply not included in the analysis of cometary emission. So, the fact is, we could have a problem. Their abundances are lower, but the details need to be checked.

The anticipated distribution of interplanetary $\mathrm{x}$-ray emission would probably resemble the distribution of scattered solar Lyman $\alpha$ off the same interstellar stream, a broad feature in the upwind direction which, if I remember correctly, shows parallax during the course of the year. It is a direction which has posed some problems in the past; roughly speaking it is difficult to see white dwarfs in the EUV in that direction (Barber et al., 1993) suggesting that absorbing interstellar material is not far away, leading us to wonder how there could be enough room to achieve the observed $x$-ray emission from the Local Bubble. Perhaps it has ultra-very local help. In the downwind direction there is a gravitationally focused column of interstellar helium which could similarly have an x-ray glow.

The material in the previous few paragraphs is new and tantalizing. Brief and exciting conversations with Walt Harris, Wilt Sanders, Ron Reynolds, Frank Scherb, and Dan McCammon contributed to its flavor, but much more thorough modeling will be needed to sort it out. It was fun, in any case, to consider yet another way in which a Breitschwerdt-and-Schmutzler-like notion may have some relevance to the SXRB.

Acknowledgements. This work was supported in part by NASA grants NAG53155 , and NAG5-629. The manuscript benefitted greatly from suggestions made by Dan McCammon, Franz Kahn, Dieter Breitschwerdt, and Bob Benjamin.

\section{References}

Arnaud, M., Rothenflug, R. and Rocchia, R. (1984): in The Local Interstellar Medium, IAU Colloq. No. 81, eds. Y. Kondo, F.C. Bruhweiler, and B.D. Savage, p. 301, NASA CP-2345

Breitschwerdt, D. \& Schmutzler, T. (1994): Nature, 371, 774

Barber, C.R., Warwick, R.S., Hodgkin, S.T., \& Pye, J.P. (1993): MNRAS, 262, 289

Bochkarev, N.G. (1987): Astrophys. Space Sci., 138, 229

Cox, D.P. (1996a): in Astrophysics in the Extreme Ultraviolet, eds. S. Bowyer and R.F. Malina, Kluwer, p. 247

Cox, D.P. (1996b): in The Interplay Between Massive Star Formation, the ISM and Galaxy Evolution, eds. D. Kunth, B. Guideroni, M. Heydari-Malayeri, and T.X. Thuan, Editions Frontieres, p. 233 
Cox, D.P. \& Anderson, P.R. (1982): ApJ, 253, 268

Cox, D.P. \& Reynolds, R.J. (1987): ARA\&A, 25, 303

Cox, D.P. \& Smith, B.W. (1974): ApJL, 189, L105

Cox, D.P. \& Snowden, S.L. (1986): Adv. Space Res., 6, 97

Cravens, T.E. (1997): Geophys. Res. Lett., 24, 105

Edgar, R.J. (1986): ApJ, 308, 389

Edgar, R.J. \&Cox, D.P. (1993): ApJ, 413, 190

Freyberg, M.J. (1997): this volume

Frisch, P. (1997) this volume

Haberli, R.M., Gombosi, T.I. De Zeeuw, D.L. Combi, \& Powell, K.G. (1997): Science, 276,939

He, X., Kerr, R.B., Bishop, J, \& Tepley, C.A. (1993): JGR, 98, p. 21, 611

Innes, D.E. \& Hartquist, T.W. (1984): MNRAS, 209,7

Jakobsen, P. \& Kahn, S.M. (1986): ApJ, 309, 682

Kahn, S.M. \& Jakobsen, P. (1988): ApJ, 329, 406

McKee, C.F. \& Ostriker J.P. (1977): ApJ, 218, 148

Shelton, R.L. (1997): this volume

Smith, R.K. (1995) PhD thesis, University of Wisconsin-Madison

Smith, R.K. and Cox, D.P. (1997): this volume

Snowden, S.L. (1997): this volume

Snowden, S.L., Egger, R., Finkbeiner, D., Freyberg, M.J. \& Plucinsky, P.P. (1997): ApJ, in press

Wang, Q.D. (1997): this volume 